LA W RENCE LIVERMORE NATIONAL LABORATORY

\section{Microstructural Characterization of Nodular Ductile Iron}

H. K. Springer

January 5, 2012 
This document was prepared as an account of work sponsored by an agency of the United States government. Neither the United States government nor Lawrence Livermore National Security, LLC, nor any of their employees makes any warranty, expressed or implied, or assumes any legal liability or responsibility for the accuracy, completeness, or usefulness of any information, apparatus, product, or process disclosed, or represents that its use would not infringe privately owned rights. Reference herein to any specific commercial product, process, or service by trade name, trademark, manufacturer, or otherwise does not necessarily constitute or imply its endorsement, recommendation, or favoring by the United States government or Lawrence Livermore National Security, LLC. The views and opinions of authors expressed herein do not necessarily state or reflect those of the United States government or Lawrence Livermore National Security, LLC, and shall not be used for advertising or product endorsement purposes.

This work performed under the auspices of the U.S. Department of Energy by Lawrence Livermore National Laboratory under Contract DE-AC52-07NA27344. 


\title{
Microstructural Characterization of Nodular Ductile Iron
}

\author{
H. K. Springer
}

\section{Summary}

The objective of this study is to quantify the graphite particle phase in nodular ductile iron (NDI). This study provides the basis for initializing microstructure in direct numerical simulations, as part of developing microstructure-fracture response models. The work presented here is a subset of a PhD dissertation on spall fracture in NDI [1]. NDI is an ideal material for studying the influence of microstructure on ductile fracture because it contains a readily identifiable second-phase particle population, embedded in a ductile metallic matrix, which serves as primary void nucleation sites. Nucleated voids grow and coalesce under continued tensile loading, as part of the micromechanisms of ductile fracture, and lead to macroscopic failure. For this study, we used 2D optical microscopy and quantitative metallography relationships to characterize the volume fraction, size distribution, nearestneighbor distance, and other higher-order metrics of the graphite particle phase. We found that the volume fraction was $\Phi=0.115$, the average particle diameter was $d_{\text {avg }}=25.9 \mu \mathrm{m}$, the Weibull shape and scaling parameters were $\beta=1.8$ and $\eta=29.1 \mu \mathrm{m}$, respectively, the (first) nearest neighbor distance was $\mathrm{L}_{n n}=32.4 \mu \mathrm{m}$, the exponential coefficients for volume fraction fluctuations was $\mathrm{A}_{\Phi}=1.89$ and $\mathrm{B}_{\Phi}=-0.59$, respectively. Based on reaching a coefficient-of-variation (COV) of 0.01 , the representative volume element (RVE) size was determined to be $8.9 \mathrm{Lnn}(288 \mu \mathrm{m})$.

\section{Methods}

\section{Material-Nodular Ductile Iron}

Nodular ductile iron (NDI) is a Fe-C-Si alloy and its chemical composition by weight is 93.3\% Fe, 3.7\% C, 2.5\% Si, 0.3\% Mn (trace amounts of $\mathrm{P}, \mathrm{S}, \mathrm{Cr}$, and $\mathrm{Mg}$ ). NDI comprises a ductile matrix (first phase) with loosely-bound, graphite particles (second-phase) that are central to its ductile fracture response [2-4]. Figure $\mathbf{1}$ depicts the two-phase microstructure of NDI in a undeformed metallographic section and in a fractograph. The graphite phase is readily identifiable in Figure 1a as the darker particles within the lighter matrix phase. The large, nearly spherical particles make NDI well-suited for quantitative metallography studies. Figure 1b clearly demonstrates that the voids nucleate at the 
graphite particles, signifying their central role in NDI ductile fracture. The material in this study met ASTM A536, 60-40-18 specifications (minimum $60 \mathrm{ksi}$ ultimate strength, minimum 40 ksi yield strength, $18 \%$ elongation in 2 inch bar) and was purchased from $\operatorname{DuraBar}^{\circledR}$.

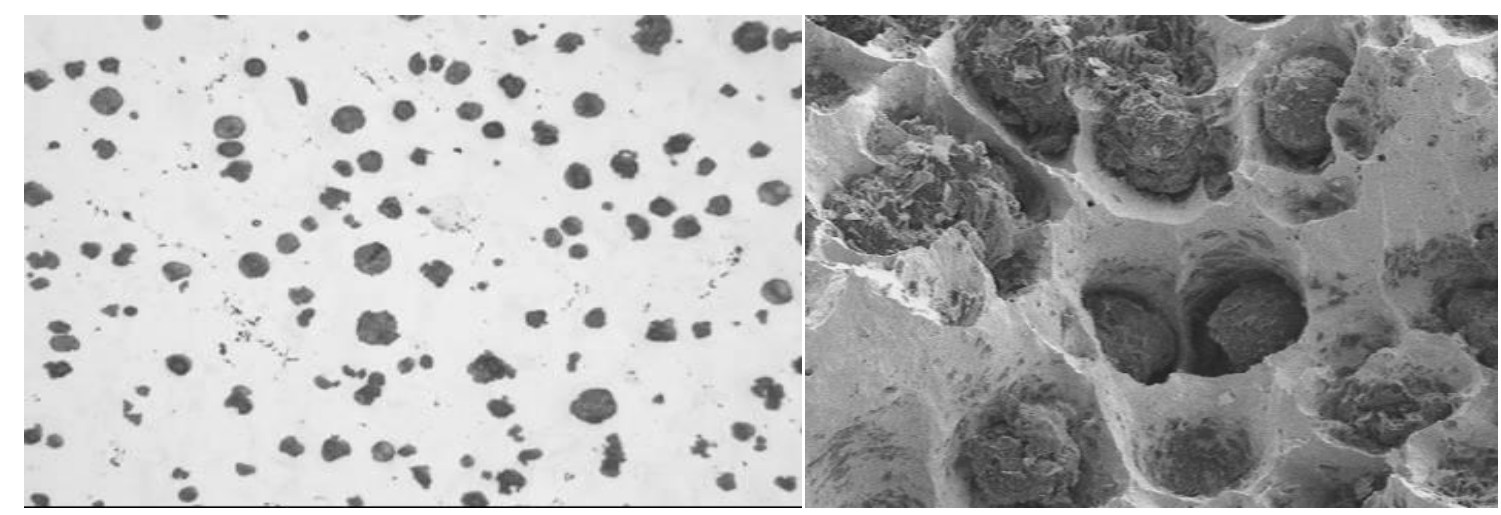

Figure 1: a. NDI two-phase microstructure consisting of graphite particles (dark) within a Fe-Si matrix (white). b. NDI fractograph demonstrating central role of graphite particles in void nucleation.

\section{Optical Microscopy}

Particle structure characterization was performed on a metallographic section of undeformed NDI. The specimen was taken from the mid-radius of the as-received NDI round stock, following the process for specimen preparation described in ASTM E-8 Standard Test Methods For Tension Testing of Metallic Materials. The mounted specimen was hand ground on successively finer SiC-impregnated polishing papers, starting with 320-grit and finishing with 4000-grit paper. The specimen was then promptly polished on a Struers TegraPol using a nylon cloth with an aqueous solution of $3 \mu \mathrm{m}$ diamond particles, washed with soap and water, and rinsed in flowing water. The specimen was then polished with $1 \mu \mathrm{m}$ diamond on a nap cloth, washed and rinsed. The specimen was then given a final polish on a nap cloth with an aqueous solution of $0.04 \mu \mathrm{m}$ colloidal silica, washed with water, rinsed with ethanol, and dried. The specimen was not etched.

A Reichert-Jun MeF3 inverted optical microscope with an automated two-axis stage was used to capture and montage 150 high resolution two dimensional images. Approximately $70,000,000 \mu \mathrm{m}^{2}$ of metallographic section data were captured at a resolution of 1.36 $\mu \mathrm{m} /$ pixel. Quantitative metallography analyses based on large image montages of the twodimensional metallographic sections has been shown to be more representative of the 
particle structure than limited, high-magnification sampling $[5,6]$. While the graphite phase is clearly recognizable in these images, further enhancements to the image contrast were necessary prior to raw data extraction. Image Pro Plus ${ }^{\circledR}$ software was used to enhance the image montage and to expedite the particle structure analysis process. The image gamma value was reduced from 1.0 to 0.5 and the contrast was increased from 50 to 80, effectively binarizing the image. An automated counting feature of Image Pro Plus was then used to obtain centroid position and mean diameter of all identified particles. These particle structure data were exported as an ASCII text file for subsequent analysis.

\section{Particle Field Analysis}

The particle area fraction is a key scalar descriptor of the particle structure that is derived from the fundamental particle size and spacing distributions. The particle area fraction was calculated from the particle structure data over the entire montage by taking the sum of the individual particle areas divided by the total area over which measurements were taken,

$$
\Phi=\frac{\sum_{i=1}^{N_{t o t}} A_{i}}{A_{t o t}}
$$

1.

where $\Phi$ is the macroscopic particle area fraction, $\mathrm{N}_{\text {tot }}$ is the total number of particles over the entire image montage, $A_{i}$ is the area of the current particle, and $A_{\text {tot }}$ is the total measurement area of the entire montage $\left(70,000,000 \mu \mathrm{m}^{2}\right)$. Since the particle area fraction was calculated over the entire image montage, it is referred to as the macroscopic particle area fraction. In statistical terms, $\Phi$ is the mean particle area fraction of the entire population. This is an important clarification, as $\Phi$ is used to develop the volume fraction fluctuation methods.

In order to determine the three-dimensional particle size distribution, it was necessary to transform the two-dimensional particle size distribution using the process described by Saltykov [7]. The transformation requires binning of the two-dimensional particle size data. The transformation equations used for this process are,

$$
\begin{aligned}
& N_{V, i}=\frac{1}{D_{i}} \sum_{j=1}^{i} N_{A, j} C_{j} \\
& C_{j}^{T}=[1.6461,-0.4561,-0.1162,-0.0415,-0.0173,-0.0079]
\end{aligned}
$$


where $\mathrm{N}_{\mathrm{V}, \mathrm{i}}$ is the ith volume bin count, $\mathrm{N}_{\mathrm{A}, \mathrm{j}}$ is the $j$ th area bin count, and $\mathrm{C}_{\mathrm{j}}$ is the Saltykov transformation constants. Based on the use of six bins in this study, $D_{i}$, the ith (two dimensional) bin diameter, was calculated with,

$$
D_{i}=\frac{1}{2}\left(\frac{D_{\max }}{6}\right)+\left(\frac{(i-1) D_{\max }}{6}\right)
$$

4.

where $D_{\max }$ is the maximum measured particle diameter. The count for each area bin, $N_{A, j}$, was determined using a simple binning algorithm on the two-dimensional particle structure data. The data has been sorted into six different bins ranging in average size from 17.3 to $104.2 \mu \mathrm{m}$, with a bin extent of approximately $17 \mu \mathrm{m}$.

The nearest neighbor distance is important for developing a relevant microstructural length-scale. Since no methods exist for transforming two-dimensional nearest neighbor distance distributions to a three-dimensional quantity, a relationship based on Corti et al. [8] was used to determine the three-dimensional nearest neighbor distance from the particle volume fraction. The relationship for nearest neighbor distance is,

$$
L_{n n}=r\left(\frac{\exp (8 \Phi)}{3 \Phi^{\frac{1}{3}}}\right) \Gamma\left(\frac{1}{3}, 8 \Phi\right)
$$

5.

where $L_{n n}$ is the average three-dimensional edge-edge nearest neighbor distance, $r$ is the average particle radius, $\Phi$ is the macroscopic particle volume fraction, and $\Gamma$ is the gamma distribution function. This relationship assumes a monodispersed system of spheres, i.e., randomly positioned but with uniform size. The graphite particles in NDI are actually a polydispersed system of spheres, i.e., a system of randomly and isotropically positioned spheres with a measurable size distribution. This assumption is inconsequential because $\mathrm{L}_{n n}$ is used to normalize the sampling size in calculating the volume fraction fluctuations, but not as a stand-alone microstructural metric.

\section{Scale-Dependent Volume Fraction Fluctuations}

Particle area and volume fraction fluctuations represent a higher order particle structure metric that provides a measure of the spatial dispersion, e.g., clustering, of the particle area and volume fraction with length-scale. Quintanilla et al. [9] and Torquato [10] defined the particle volume fraction fluctuations as the particle volume fraction coefficient-of-variation (COV). In their definition of the COV, the standard deviation of the particle volume fraction 
sample ensemble is divided by the macro-scopic particle volume fraction i.e., the mean particle volume fraction of the entire particle population. A multiscale sampling technique was developed here to statistically determine the length-scale dependent area fraction fluctuations from the particle structure data. This technique is illustrated in Figure 2, where an ensemble of equally-sized sampling windows is generated and centered at random locations in the particle structure domain.

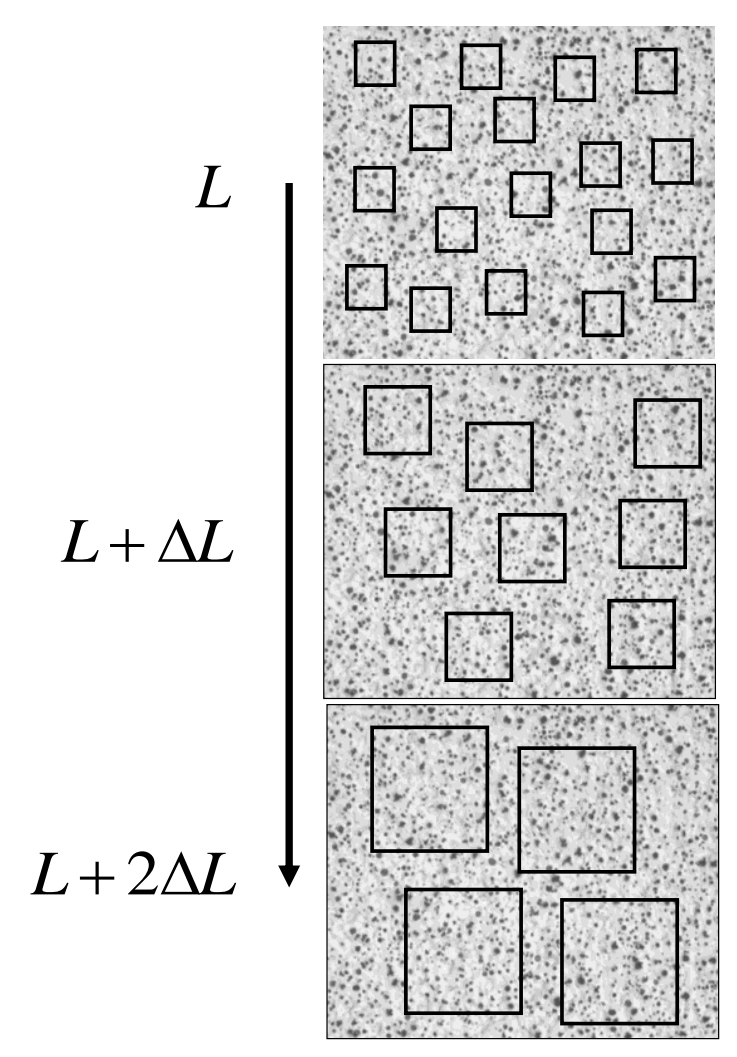

Figure 2: Multiscale sampling technique for determining particle area fraction fluctuations in a metallographic section of undeformed NDI. Incrementally larger sampling window sizes are shown.

The particle area fraction, $\varphi_{\mathrm{i}}$, of the $i t h$ sampling window was determined, 


$$
\phi_{i}\left(L_{k}\right)=\frac{\sum_{j=1}^{N_{\text {paricles }}} A_{\text {part }, j}}{L_{k}^{2}}
$$

where $L_{k} \times L_{k}$ is the area of the (square) sampling window, $A_{\text {part,j }}$ is the area of the $j$ th particle and there are $\mathrm{N}_{\text {particles }}$ in the sampling window. The sampling ensemble consists of $\mathrm{N}_{\text {samples }}$ total windows. The subscript $k$ refers to the $k t h$ window size. Equation (1) is calculated over the entire image montage, whereas equation (6) is calculated over the sampling regions (subsets) of the image montage. As part of determining the COV, the standard deviation, $S_{\varphi}\left(L_{k}\right)$, over the entire sample ensemble is calculated,

$$
\begin{aligned}
& S_{\phi}\left(L_{k}\right)=\sqrt{\frac{\sum_{i=1}^{N_{\text {samples }}}\left(\phi_{i}\left(L_{k}\right)-\Phi\right)^{2}}{N_{\text {samples }}}} \\
& \operatorname{COV}_{\phi}\left(L_{k}\right)=\frac{S_{\phi}\left(L_{k}\right)}{\Phi}
\end{aligned}
$$

7.

8.

where $S_{\varphi}\left(L_{k}\right)$ is the standard deviation of the particle area fractions for a fixed window size (fixed $L_{k}$ ), $N_{\text {samples }}$ is the total number of samples (fixed at 100 samples), $\Phi$ is the macroscopic particle area fraction, and $\operatorname{COV}_{\varphi}$ is the particle area fraction COV at the fixed window size. Quintanilla et al. [9] and Torquato [10] used $\Phi$ in the calculation of $\operatorname{COV}_{\varphi}$ because it simplifies arguments for convergence with increased window size, i.e., as the length-scale becomes large, the COV approaches zero. Torquato [10] argued that normalization with the sample mean, rather than the "true" mean of the entire particle population, would not necessarily guarantee convergence with increased sampling window size. As shown in Figure 2, this analysis was repeated for a range of sampling window sizes, $\mathrm{L}_{0}, \mathrm{~L}_{0}+\Delta \mathrm{L}, \mathrm{L}_{0}+2 \Delta \mathrm{L}$, where $\mathrm{L}_{0}$ is $25 \mu \mathrm{m}$ and $\Delta \mathrm{L}$ is $25 \mu \mathrm{m}$.

As with the 1:1 transformation between particle area and volume fractions for a polydispersed system of spherical particles, it was assumed that a 1:1 transformation also applies for area fraction fluctuations on a sample-per-sample basis. Therefore, the nondimensional particle area fraction COV measurements above were treated as particle volume fraction fluctuations. Random positioning of sampling windows reflected the uncorrelated nature of the NDI particle size and spacing distributions. This is particular true at the smallest window sizes considered in this study. Since the NDI second-phase particles are assumed to be isotropic, the directionality was not considered in the sampling process. 


\section{Results}

The particle area fraction, $\Phi$, was found to be 0.115 . Since the particle area fraction and the volume fraction are identical for a polydispersed system of spheres [8,11], the macroscopic particle volume fraction, $\Phi$, is also 0.115 .

As shown in Figure 3, a two parameter Weibull probability distribution function (PDF) with lower $(17 \mu \mathrm{m})$ and upper $(100 \mu \mathrm{m})$ particle size bounds was used to fit the (Saltykovtransformed) three-dimensional particle diameter, D, data,

$$
P(D)=\frac{\beta D^{\beta-1}}{\eta^{\beta}} \exp \left(-\left(\frac{D}{\eta}\right)^{\beta}\right)
$$

where the fitted Weibull shape and scaling parameters are: $\beta=1.8$ and $\eta=29.1 \mu \mathrm{m}$, respectively. The mean three-dimensional particle diameter is $25.9 \mu \mathrm{m}$. Based on the measured average particle diameter and the macroscopic particle volume fraction, the nearest neighbor distance, $\mathrm{L}_{\mathrm{nn}}$, is calculated to be $32.4 \mu \mathrm{m}$.

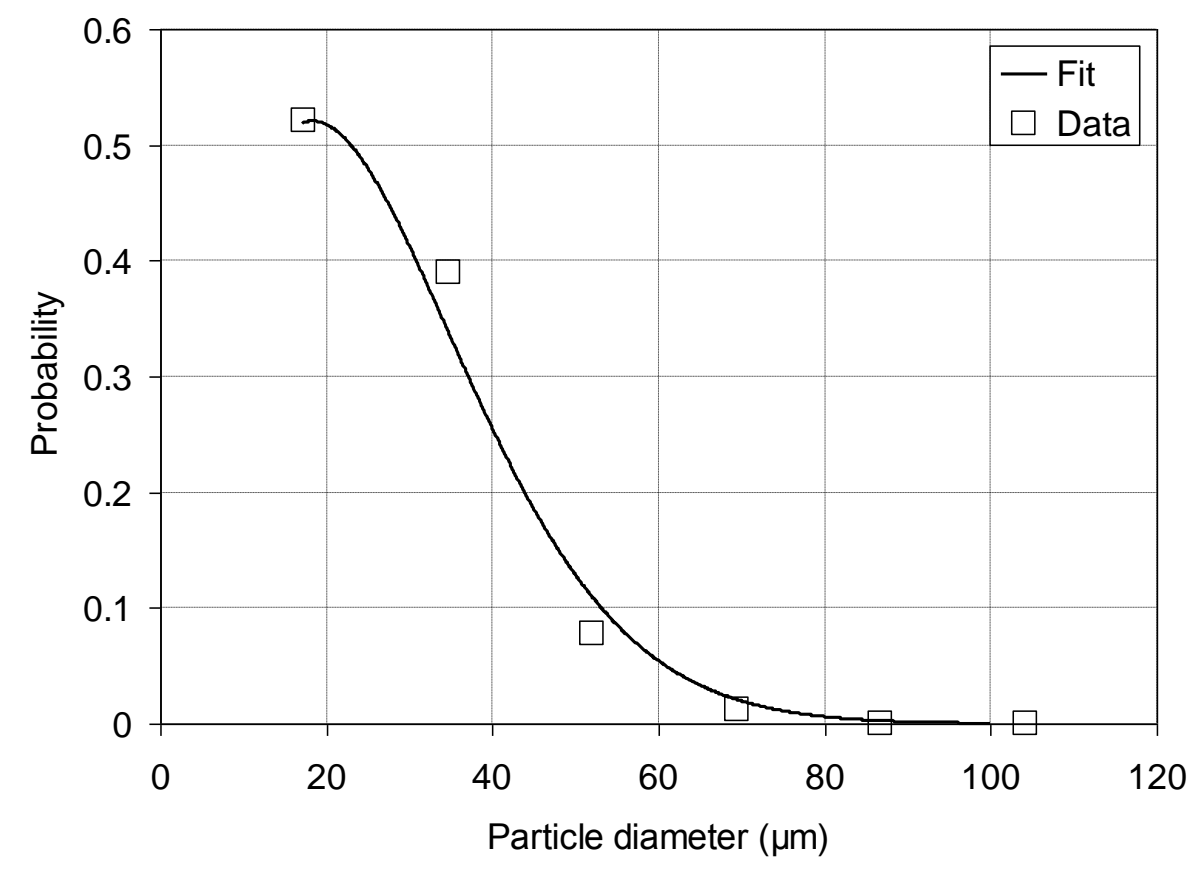

Figure 3: Three-dimensional particle diameter probability distribution function (PDF) plots for the binned test data and corresponding Weibull PDF fit. 
As with particle area and volume fractions, the area and volume fraction fluctuations were assumed to be identical. As shown in Figure 4, the particle volume fraction fluctuation metric, $\operatorname{COV}_{\varphi}(\mathrm{L})$, was observed to decrease with increasing normalized sampling size (L/ $\mathrm{Lnn}_{\mathrm{n}}$ ) and an exponential function adequately describes the decay,

$$
\operatorname{COV}_{\phi}(L)=\left[A_{\phi} \exp \left(B_{\phi} \frac{L}{L_{n n}}\right)\right]
$$

10.

where $\mathrm{L}$ is the (variable) sampling size, and $\mathrm{L}_{\mathrm{nn}}$ is the nearest neighbor distance (32.4 $\left.\mu \mathrm{m}\right)$. The exponential coefficients are $A_{\Phi}=1.89$ and $B_{\Phi}=-0.59 . A_{\Phi}, B_{\Phi}$, and $L_{n n}$ are unique to the graphite particle structure in NDI. In order to establish a notional representative volume element (RVE) size in NDI, $\operatorname{COV}_{\varphi}(\mathrm{L})$ was specified to be 0.01 . This value essentially means that the fluctuations are fixed at $1 \%$ of the mean volume fraction. At this value, the RVE size was extrapolated to be $8.9 \mathrm{Lnn}(288 \mu \mathrm{m})$.

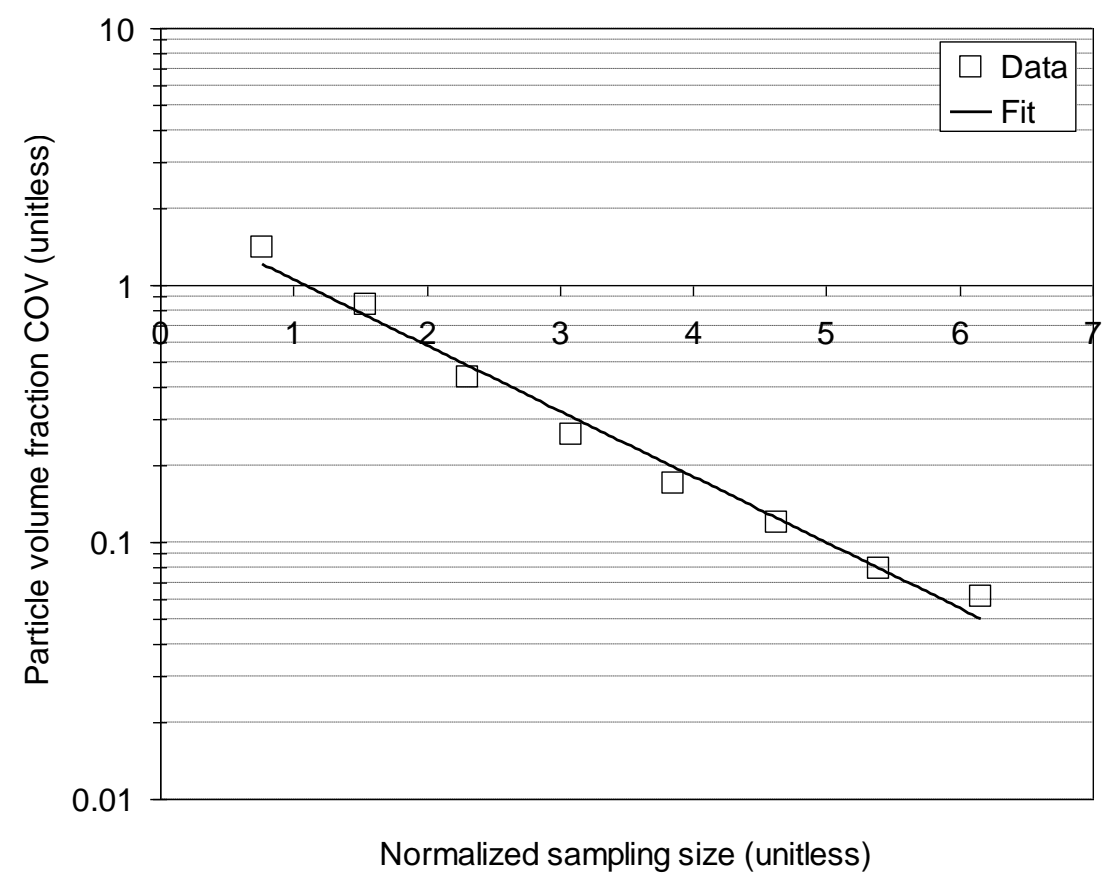

Figure 4: Particle volume fraction COV as a function of normalized sampling size $\left(L / L_{n n}\right)$

An analytical model for the heterogeneous second-phase particle structure in NDI was developed. A Gaussian PDF was fit to the length-scale dependent particle volume fraction using the standard deviation in equation (11) and $\Phi$, 


$$
P_{\phi}(\phi, L)=\frac{1}{S_{\phi}(L) \sqrt{2 \pi}} \exp \left(\frac{-1}{2}\left[\frac{\phi-\Phi}{S_{\phi}(L)}\right]^{2}\right)
$$

where $\mathrm{P}_{\varphi}(\varphi, \mathrm{L})$ is the particle volume fraction $\mathrm{PDF}$ and $\varphi$ is the (variable) local particle volume fraction at length-scale, L. Figure 5 illustrates the change in $\mathrm{P}_{\varphi}(\varphi, \mathrm{L})$ with varying length-scale. At $\mathrm{L}=6 \mathrm{~L}_{\mathrm{nn}}$ to $8 \mathrm{~L}_{\mathrm{nn}}$, the standard deviation decreases and as $\mathrm{L} \rightarrow \infty$, then $\mathrm{S}_{\varphi} \rightarrow$ 0 , with $\mathrm{P}_{\varphi}(\varphi, \mathrm{L})$ essentially becoming a single-valued function $(\varphi \rightarrow \Phi)$. Alternately, the spread in $\mathrm{P}_{\varphi}(\varphi, \mathrm{L})$ increases with decreasing length-scale, $\mathrm{L} \rightarrow \mathrm{L}_{n n}$, and the probability of sampling $\Phi$ is not substantially greater than larger or smaller values.

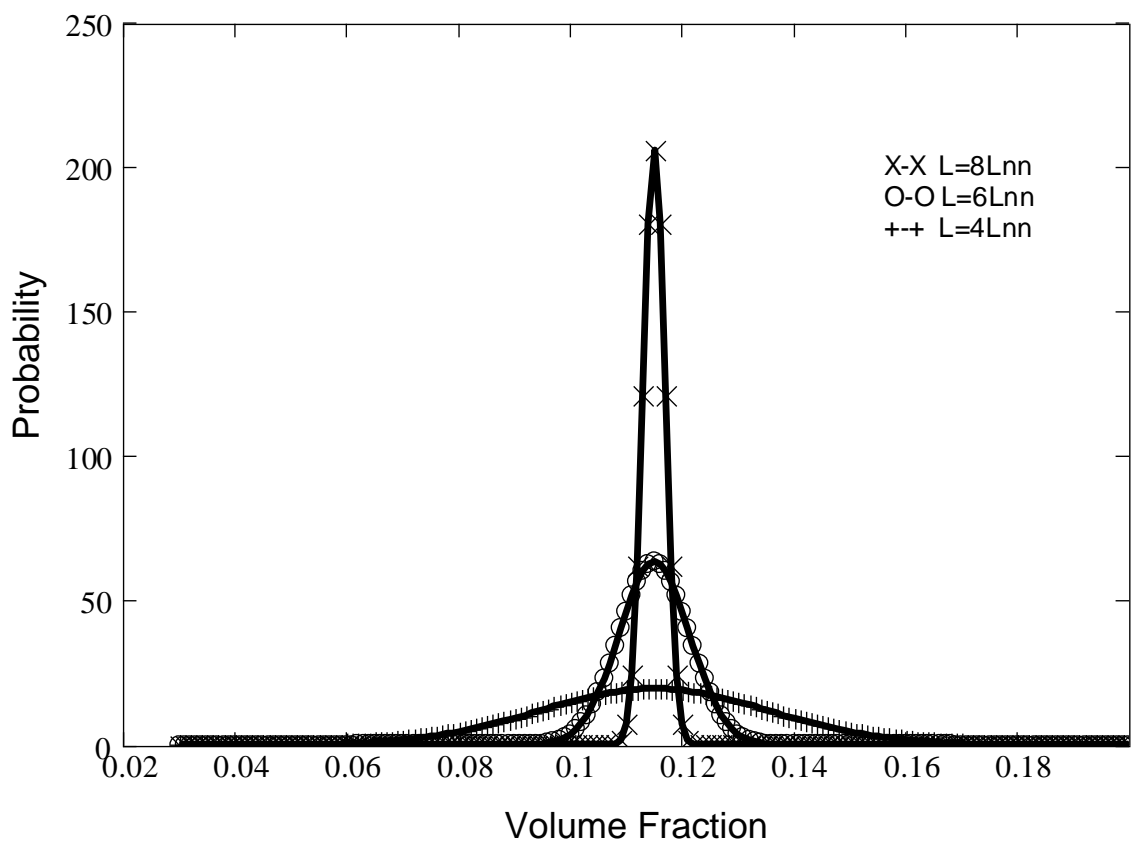

Figure 5: $P_{\varphi}(\varphi, L)$ as a function of length-scale

\section{Discussion}

In light of equation (11), the NDI particle volume fraction field, $\varphi(\mathrm{x}, \mathrm{L})$, can be regarded as a superposition of fluctuations, $\varphi^{*}$, onto a mean field, $\Phi$ :

$$
\phi(x, L)=\Phi+\phi^{*}(x, L)
$$


Here the spatial variability and length-scale dependence in the particle volume fraction field, $\varphi(\mathrm{x}, \mathrm{L})$, is due entirely to the fluctuating component, $\varphi^{*}(\mathrm{x}, \mathrm{L})$. While $\Phi$ is the integral of the local particle volume fraction over the large three-dimensional domain, it was approximated by taking the particle area fraction over the entire image montage. In general, the fluctuating component of the particle volume fraction field is both dependent on location and length-scale over which it is measured, i.e., $\varphi(\mathrm{x}, \mathrm{L})$. Since we have assumed the particle field to be isotropic and spatially random (spatially uncorrelated), only the length-scale dependence is explicitly considered for the fluctuating component, i.e., $\varphi^{*}(\mathrm{x}, \mathrm{L})$ $\approx \varphi^{*}(\mathrm{~L})$. Consistent with this notion, the multiscale sampling technique used for acquiring particle volume fraction fluctuation data did not record sample position or orientation data. These particle volume fraction fluctuation measurements form the basis of $\mathrm{P}_{\varphi}(\varphi, \mathrm{L})$.

The RVE in materials with heterogeneous particle structures, such as NDI, go beyond unit cell notions. RVE size is a variable quantity based on property measured and is much larger than characteristic material length-scales. Hill [12] and other investigators [13,14] have suggested that a RVE in realistic materials enclose a sufficiently large number of heterogeneities to adequately capture a macroscopically uniform stress or strain response under uniform macroscopic loading. Based on this principle, we established a notional RVE size in NDI by specifying $\operatorname{COV}_{\varphi}(\mathrm{L})$ to be 0.01 . This value essentially means that the fluctuations are $1 \%$ of the mean volume fraction. This corresponded to an RVE size of $8.9 \mathrm{Lnn}_{\mathrm{nn}}(288 \mu \mathrm{m})$, which informs setup of direct numerical simulations. However, we cannot expect that the constitutive behavior is solely regulated by the graphite particle phase, i.e., the RVE for deformation and fracture response is only influenced by the particle volume fraction COV. Moreover, fracture is a localized phenomenon and may be more related to particle clustering in the material and other concentrated regions of particles. Direct numerical simulations in other studies are improving our understanding of the role of microstructure in NDI fracture response [1].

\section{Acknowledgements}

Special thanks to Tom Mason of Los Alamos National Laboratory for providing their automated optical microscopy facilities and to Jackson Go of Lawrence Livermore National Laboratory for helping prepare metallographic specimens. This work was performed under the auspices of the U.S. DOE by LLNL under Contract DE-AC52-07NA27344. This research was supported by the Joint DoD-DOE Munitions Technology Development Program. 


\section{References}

1. Springer, H. K., Mesoscale Modeling of Spall in a Heterogeneous Two-Phase Material, Ph.D. dissertation, UC Davis (2008)

2. Clough, WR, Shank, ME; The Flow and Fracture of Nodular Cast Iron; Trans. Am. Mech. Engrs., 79 (1957) pp 1911-1920

3. Shi, J, Savas, MA, Smith, RW; Plastic deformation of a model material containing soft spheroidal inclusions: Spheroidal graphite cast iron; Journal of Materials Processing Technology, 133 (2003) pp 297-303

4. Liu, JH, Hao, $\mathrm{XY}, \mathrm{Li}, \mathrm{GL}, \mathrm{Liu}, \mathrm{GS}$; Microvoid evaluation of ferrite ductile iron under strain; Materials Letters 56 (2002) pp 748-755

5. Shan, Z, Gokhale, AM; Representative Volume Element for Non-Uniform Microstructure; Computational Materials Science, 24 (2002) pp 361-379

6. Yang, S, Tewari, A, Gokhale, AM; Modeling of non-uniform spatial arrangement of fibers in a ceramic matrix composite; Acta mater. $45: 7$ (1997) pp 3059-3069

7. Saltykov, SA; Developments in Stereology, H. Elias, Ed., Proceedings of the Second International Congress for Stereology, Springer-Verlag, New York (1967) p 163

8. Corti, CW, Cotterill, P, Fitzpatrick, GA; The Evaluation of the Interparticle Spacing in Dispersion Alloys; International Metallurgical Reviews, 19 (1974) pp 77-88

9. Quintanilla, J, Torquato, S; Local volume fraction fluctuations in random media; J. Chem. Phys. 106:7 (1997) pp 2741-2751

10. Torquato, S; Random Heterogeneous Materials: Microstructure and Macroscopic Properties; Springer-Verlag (2002)

11. Underwood, EE; The mathematical foundations of quantitative stereology; Stereology and Quantitative Metallography, ASTM STP 504, ASTM (1972) pp 3-38

12. Hill, R; Journal of the Mechanics and Physics of Solids, 11 (1963) pp 357-372

13. Hashin, Z, Shtrikman, S; J. Mech. Phys. Solids, 10 (1962) p 335

14. Nemat-Nasser, S, Hori, M; Micromechanics: Overall properties of heterogeneous materials; North-Holland Elsevier, $2^{\text {nd }}$ Edition (1999) 